\title{
Editorial: Metformin: Beyond Diabetes
}

\author{
Frédéric Bost ${ }^{1 *}$, Graham Rena ${ }^{2 *}$ and Benoit Viollet ${ }^{3 *}$ \\ ${ }^{1}$ Université Nice Côte d'Azur, Inserm U1065, Nice, France, ${ }^{2}$ University of Dundee, Dundee, United Kingdom, ${ }^{3}$ Université de \\ Paris, Institut Cochin, CNRS UMR8104, INSERM U1016, Paris, France
}

Keywords: metformin, cancer, diabetes, neurodegenerative disease, mitochondria, gut microbiota, PCOS (polycystic ovarian syndrome)

\section{Editorial on the Research Topic}

\section{Metformin: Beyond Diabetes}

Metformin, a member of the family of biguanides, is one of the most prescribed medications in the US and Europe and remains the first-line treatment for type 2 Diabetes (T2D) worldwide. It is a low cost medication, relatively well-tolerated, that has been given to millions of patients for more than 60 years in Europe. The literature on metformin is immense and recent discoveries in basic research place metformin on the short-list of the most promising drug for repurposing. Pioneering mechanistic studies demonstrating that metformin inhibits complex I in the respiratory chain of the mitochondria $(1,2)$ and the work of Zhou et al. showing that metformin activates AMP-activated protein kinase (AMPK) by inducing its phosphorylation at $\operatorname{Thr}^{172}$ (3), opened new horizons

OPEN ACCESS

Edited and reviewed by: Ralf Jockers, Université Paris-Sorbonne, France

*Correspondence: Frédéric Bost bost@unice.fr Graham Rena g.rena@dundee.ac.uk Benoit Viollet benoit.viollet@inserm.fr

Specialty section:

This article was submitted to Cellular Endocrinology, a section of the journal Frontiers in Endocrinology

Received: 15 November 2019 Accepted: 21 November 2019 Published: 06 December 2019

Citation:

Bost F, Rena $G$ and Viollet B (2019) Editorial: Metformin: Beyond Diabetes.

Front. Endocrinol. 10:851.

doi: 10.3389/fendo.2019.00851 for maximizing clinical exploitation of metformin. Not only did they spur better understanding of metformin's action in T2D (4-6), but they also provided rational bases for laboratories to study the therapeutic potential of metformin outside of the conventional management of T2D. Twenty years on however, there still remains much debate regarding the key molecular target(s) of metformin. In this Research Topic, the evidence regarding direct effects of metformin on complex I of the electron transport chain and mitochondria are discussed in two focussed reviews (Fontaine; Vial et al.). They address topical research alongside earlier studies on the mechanism of action of metformin on mitochondrial complex I, how metformin modulates reactive oxygen species (ROS) production to prevent mitochondrial-mediated apoptosis and how the drug protects against permeability transition pore (PTP)-induced cell death. These effects are discussed in the context of $\mathrm{T} 2 \mathrm{D}$ and cancer.

Metformin is now a well-established disruptor of cellular energy supply that targets the mitochondria [(7); Fontaine; Vial et al.]. The resulting compensatory changes on cellular metabolism to provide alternative sources of ATP and metabolites are detailed in this Research Topic by Andrzejewski et al.: including increased glycolysis, modifications of glutamine metabolism, and increase in PGC- $1 \alpha$ [a major regulator of mitochondrial biogenesis also implicated in cancer (8)]. These adaptations are thought to play a central role in the resistance to metformin in cancer cells.

Activation of AMPK has been reported to inhibit the mechanistic target of rapamycin complex 1 (mTORC1) signaling pathway frequently activated in cancer cells (9). Furthermore, the tumor suppressor LKB1 was demonstrated to phosphorylate AMPK in response to biguanides (10). What then are the consequences in terms of cancer incidence in patients treated with metformin for decades? Observational evidence suggests that metformin reduces the incidence of cancer in people with diabetes (11). In this Research Topic three articles focus on the action of metformin on cancer 
and more specifically on melanoma (Jaune and Rocchi), leukemia (Biondani and Peyron), and colorectal cancer (Higurashi and Nakajima). These reviews describe in detail the recent advances concerning in vivo effects and the different molecular mechanisms underlying the anti-cancer action of metformin (AMPK dependent/independent effects, role of p53 and cellular effects: apoptosis, autophagy, proliferation, and cell migration), and present ongoing clinical trials for the prevention or treatment of various types of cancer.

One of the first reported benefits of metformin in reproductive biology was the increase of fertility in patients with polycystic ovary syndrome (PCOS) (12). This pathology is often associated with insulin resistance; thus, it is perhaps not surprising in hindsight that metformin ameliorates PCOS. Likewise, metformin has beneficial effects on obese male fertility (13). This important aspect of metformin action is addressed in a review that also discusses the potential epigenetic modifications induced by metformin in this context (Faure et al.). Among epigenetic modifications, histone acetylation/deacetylation plays a major role in the regulation of gene expression and metformin via AMPK was shown to regulate the expression of Sirtuin1 (Sirt1), a member of the class III (NAD+dependent) histone deacetylases (HDACs) (14). An original research article of this collection by the group of J. Menendez, uses a computational approach to identify putative sites of interaction between Sirt1 and metformin (Cuyàs et al.). This is an important issue since metformin similarly to Sirtuins has been reported to expand longevity from yeast to mammals (15).

One of the most surprising effects of metformin found in recent years is its action on the gut microbiota. Indeed, the original discovery made by Oluf Pedersen's lab demonstrated that

\section{REFERENCES}

1. El-Mir MY, Nogueira V, Fontaine E, Averet N, Rigoulet M, Leverve X. Dimethylbiguanide inhibits cell respiration via an indirect effect targeted on the respiratory chain complex I. J Biol Chem. (2000) 275:223-8. doi: $10.1074 /$ jbc. 275.1 .223

2. Owen MR, Doran E, Halestrap AP. Evidence that metformin exerts its anti-diabetic effects through inhibition of complex 1 of the mitochondrial respiratory chain. Biochem J. (2000) 348(Pt 3), 607-614. doi: 10.1042/bj3480607

3. Zhou G, Myers R, Li Y, Chen Y, Shen X, Fenyk-Melody J, et al. Role of AMPactivated protein kinase in mechanism of metformin action. J Clin Invest. (2001) 108:1167-74. doi: 10.1172/JCI13505

4. Foretz M, Guigas B, Viollet B. Understanding the glucoregulatory mechanisms of metformin in type 2 diabetes mellitus. Nat Rev Endocrinol. (2019) 15:56989. doi: 10.1038/s41574-019-0242-2

5. Foretz M, Hebrard S, Leclerc J, Zarrinpashneh E, Soty M, Mithieux G, et al. Metformin inhibits hepatic gluconeogenesis in mice independently of the LKB1/AMPK pathway via a decrease in hepatic energy state. J Clin Invest. (2010) 120:2355-69. doi: 10.1172/JCI40671

6. Madiraju AK, Qiu Y, Perry RJ, Rahimi Y, Zhang XM, Zhang D, et al. Metformin inhibits gluconeogenesis via a redox-dependent mechanism in vivo. Nat Med. (2018) 24:1384-94. doi: 10.1038/s41591-018-0125-4

7. Bost F, Decoux-Poullot AG, Tanti JF, Clavel S. Energy disruptors: rising stars in anticancer therapy? Oncogenesis. (2016) 5:e188. doi: 10.1038/oncsis.2015.46 metformin causes a shift in the composition of microbiota altered during T2D (16). Two examples of the action of metformin on gut microbiota are given in two original research papers of the "Metformin: beyond diabetes" Research Topic (Wang et al.; Ji et al.).

Finally, there is growing evidence showing that metformin may have therapeutic potential in neurodegenerative disease. Rotermund et al. contribute a comprehensive review on the topic. In this article, evidence for effects of metformin on Alzheimer's disease, Parkinson's disease, amyotrophic lateral sclerosis, and Huntington's disease are summarized. Metformin has a protective action on neurons mainly because it protects from oxidative stress and neuroinflammation through mechanisms implicating mitochondria and glucose metabolism. Once again, cellular metabolism is at the forefront.

There are so many pathologies that have been shown to be impacted by metformin that it is a first-class candidate for drug repurposing in the near future. Besides cardiovascular disease $(17,18)$, tuberculosis (19) and very recently multiple sclerosis (20) may show promise. Future investigations and large-scale prospective clinical trials, some of them currently ongoing, will clarify this fascinating issue.

\section{AUTHOR CONTRIBUTIONS}

All authors listed have made a substantial, direct and intellectual contribution to the work, and approved it for publication.

\section{FUNDING}

Research funded by Fondation ARC to FB, BHF PG/18/79/34106 and the Newton Fund to GR.
8. Kaminski L, Torrino S, Dufies M, Djabari Z, Haider R, Roustan $\mathrm{FR}$, et al. PGClalpha inhibits polyamine synthesis to suppress prostate cancer aggressiveness. Cancer Res. (2019) 79:3268-80. doi: 10.1158/0008-5472.CAN-18-2043

9. Saxton RA, Sabatini DM. mTOR Signaling in Growth, Metabolism, and Disease. Cell. (2017) 168:960-76. doi: 10.1016/j.cell.2017. 02.004

10. Hawley SA, Boudeau J, Reid JL, Mustard KJ, Udd L, Makela TP, et al. Complexes between the LKB1 tumor suppressor, STRAD alpha/beta and MO25 alpha/beta are upstream kinases in the AMP-activated protein kinase cascade. J Biol. (2003) 2:28. doi: 10.1186/1475-4924-2-28

11. Evans JM, Donnelly LA, Emslie-Smith AM, Alessi DR, Morris AD. Metformin and reduced risk of cancer in diabetic patients. BMJ. (2005) 330:1304-5. doi: 10.1136/bmj.38415.708634.F7

12. Patel R, Shah G. Effect of metformin on clinical, metabolic and endocrine outcomes in women with polycystic ovary syndrome: a meta-analysis of randomized controlled trials. Curr Med Res Opin. (2017) 33:1545-57. doi: 10.1080/03007995.2017.1279597

13. Ferreira C, Sousa M, Rabaca A, Oliveira PF, Alves MG, Sa R. Impact of metformin on male reproduction. Curr Pharm Des. (2015) 21:3621-33. doi: 10.2174/1381612821666150710150041

14. Canto C, Gerhart-Hines Z, Feige JN, Lagouge M, Noriega L, Milne JC, et al. AMPK regulates energy expenditure by modulating NAD+ metabolism and SIRT1 activity. Nature. (2009) 458:1056-60. doi: 10.1038/nature 07813 
15. Martin-Montalvo A, Mercken EM, Mitchell SJ, Palacios HH, Mote PL, Scheibye-Knudsen M, et al. Metformin improves healthspan and lifespan in mice. Nat Commun. (2013) 4:2192. doi: 10.1038/ncomms3192

16. Forslund K, Hildebrand F, Nielsen T, Falony G, Le Chatelier E, Sunagawa S, et al. Disentangling type 2 diabetes and metformin treatment signatures in the human gut microbiota. Nature. (2015) 528:262-6. doi: 10.1038/nature15766

17. Mohan M, Al-Talabany S, McKinnie A, Mordi IR, Singh JSS, Gandy SJ, et al. A randomized controlled trial of metformin on left ventricular hypertrophy in patients with coronary artery disease without diabetes: the MET-REMODEL trial. Eur Heart J. (2019) 40:3409-17. doi: 10.1093/eurheartj/ehz203

18. Rena G, Lang CC. Repurposing metformin for cardiovascular disease. Circulation. (2018) 137:422-4. doi: 10.1161/CIRCULATIONAHA.117.031735

19. Leung CC. Repurposing metformin to prevent and treat tuberculosis. Respirology. (2018) 23:974-5. doi: 10.1111/resp.13371
20. Neumann B, Baror R, Zhao C, Segel M, Dietmann S, Rawji KS, et al. Metformin restores CNS remyelination capacity by rejuvenating aged stem cells. Cell Stem Cell. (2019) 25:473-485.e478. doi: 10.1016/j.stem.2019.08.015

Conflict of Interest: The authors declare that the research was conducted in the absence of any commercial or financial relationships that could be construed as a potential conflict of interest.

Copyright (c) 2019 Bost, Rena and Viollet. This is an open-access article distributed under the terms of the Creative Commons Attribution License (CC BY). The use, distribution or reproduction in other forums is permitted, provided the original author(s) and the copyright owner(s) are credited and that the original publication in this journal is cited, in accordance with accepted academic practice. No use, distribution or reproduction is permitted which does not comply with these terms. 